\title{
An iterative method for split hierarchical monotone variational inclusions
}

\author{
Qamrul Hasan Ansari ${ }^{1,2^{*}}$ and Aisha Rehan ${ }^{1}$
}

\author{
"Correspondence: \\ qhansari@gmail.com \\ 1 Department of Mathematics, \\ Aligarh Muslim University, Aligarh, \\ 202002, India \\ ${ }^{2}$ Department of Mathematics \& \\ Statistics, King Fahd University of \\ Petroleum \& Minerals, Dhahran, \\ Saudi Arabia
}

\begin{abstract}
In this paper, we introduce a split hierarchical monotone variational inclusion problem (SHMVIP) which includes split variational inequality problems, split monotone variational inclusion problems, split hierarchical variational inequality problems, etc., as special cases. An iterative algorithm is proposed to compute the approximate solutions of an SHMVIP. The weak convergence of the sequence generated by the proposed algorithm is studied. We present an example to illustrate our algorithm and convergence result.
\end{abstract}

MSC: Primary 49J40; 47J20; secondary 49J52; 49M05

Keywords: split hierarchical monotone variational inclusions; fixed point problems; iterative method; maximal monotone set-valued mappings; resolvent operators; convergence analysis

\section{Introduction}

Let $H_{1}$ and $H_{2}$ be real Hilbert spaces, $C \subseteq H_{1}$ and $Q \subseteq H_{2}$ be nonempty, closed, and convex sets, $A: H_{1} \rightarrow H_{2}$ be a bounded linear operator, and $f: H_{1} \rightarrow H_{1}$ and $g: H_{2} \rightarrow H_{2}$ be two given operators. Recently, Censor et al. [1] introduced the following split variational inequality problem (SVIP):

$$
\text { Find } x^{*} \in C \text { such that }\left\langle f\left(x^{*}\right), x-x^{*}\right\rangle \geq 0, \quad \text { for all } x \in C \text {, }
$$

and such that

$$
y^{*}:=A x^{*} \in Q \text { solves }\left\langle g\left(y^{*}\right), y-y^{*}\right\rangle \geq 0, \quad \text { for all } y \in Q .
$$

Let $\Lambda$ denote the solution set of the SVIP, that is,

$$
\Lambda=\{x \text { solves }(1.1): A x \text { solves }(1.2)\} .
$$

If $f$ and $g$ are convex and differentiable, then the SVIP is equivalent to the following split minimization problem:

$$
\min f(x) \text {, subject to } x \in C \text {, }
$$

(c) 2015 Ansari and Rehan. This article is distributed under the terms of the Creative Commons Attribution 4.0 International License (http://creativecommons.org/licenses/by/4.0/), which permits unrestricted use, distribution, and reproduction in any medium, provided you give appropriate credit to the original author(s) and the source, provide a link to the Creative Commons license, and indicate if changes were made. 
and such that

$$
y^{*}:=A x^{*} \in Q \text { solves } \min g(y), \quad \text { subject to } y \in Q
$$

For further details on the equivalence between a variational inequality and an optimization problem, we refer to [2]. The SVIP also contains the split feasibility problem (SFP) [3] as a special case. For further details of the SFP, we refer to [4, 5] and the references therein.

If the sets $C$ and $Q$ are the set of fixed points of the operators $T: H_{1} \rightarrow H_{1}$ and $S$ : $\mathrm{H}_{2} \rightarrow \mathrm{H}_{2}$, respectively, then the SVIP is called a split hierarchical variational inequality problem (SHVIP). It is introduced and studied by Ansari et al. [6]. Several special cases of a SHVIP, namely, the split convex minimization problem, the split variational inequality problem defined over the solution set of monotone variational inclusion problem, the split variational inequality problem defined over the solution set of equilibrium problem, are also considered in [6].

Let $B_{1}: H_{1} \rightrightarrows H_{1}$ and $B_{2}: H_{2} \rightrightarrows H_{2}$ be set-valued mappings with nonempty values, and let $f: H_{1} \rightarrow H_{1}$ and $g: H_{2} \rightarrow H_{2}$ be mappings. Then, inspired by the work in [1], Moudafi [7] introduced the following split monotone variational inclusion problem (SMVIP):

$$
\text { Find } x^{*} \in H_{1} \text { such that } 0 \in f\left(x^{*}\right)+B_{1}\left(x^{*}\right) \text {, }
$$

and such that

$$
y^{*}:=A x^{*} \in H_{2} \text { solves } 0 \in g\left(y^{*}\right)+B_{2}\left(y^{*}\right) \text {. }
$$

Let $\Gamma$ denote the solution set of SMVIP, that is,

$$
\Gamma=\{x \text { solves }(1.5): A x \text { solves }(1.6)\} .
$$

To solve the SMVIP, Moudafi [7] proposed the following iterative method: Let $\lambda>0$ and $x_{0}$ be the initial guess. Compute

$$
x_{n+1}=U\left(x_{n}+\gamma A^{*}(T-I) A x_{n}\right), \quad \text { for all } n \in \mathbb{N},
$$

where $\gamma \in(0,1 / L)$ with $L$ being the spectral radius of the operator $A^{*} A, U=J_{\lambda}^{B_{1}}(I-\lambda f)$, $T=J_{\lambda}^{B_{2}}(I-\lambda g)$, and $J_{\lambda}^{B_{1}}$ and $J_{\lambda}^{B_{2}}$ are the resolvents of $B_{1}$ and $B_{2}$, respectively, with parameter $\lambda$ (see [8]). He obtained the following weak convergence result for iterative method (1.7).

Theorem 1.1 ([7], Theorem 2.1) Given a bounded linear operator $A: H_{1} \rightarrow H_{2}$. Let $f$ : $H_{1} \rightarrow H_{1}$ and $g: H_{2} \rightarrow H_{2}$ be $\alpha_{1}$ and $\alpha_{2}$ inverse strongly monotone operators on $H_{1}$ and $H_{2}$, respectively, and $B_{1}, B_{2}$ be two maximal monotone operators, and set $\alpha:=\min \left\{\alpha_{1}, \alpha_{2}\right\}$. Consider the operator $U:=J_{\lambda}^{B_{1}}(I-\lambda f), T:=J_{\lambda}^{B_{2}}(I-\lambda g)$ with $\lambda \in(0,2 \alpha)$. Then the sequence $\left\{x_{n}\right\}$ generated by (1.7) converges weakly to an element $x^{*} \in \Gamma$, provided that $\Gamma \neq \varnothing$ and $\gamma \in(0,1 / L)$.

Let $T: H_{1} \rightarrow H_{1}$ and $S: H_{2} \rightarrow H_{2}$ be operators such that $\operatorname{Fix}(T) \neq \emptyset$ and $\operatorname{Fix}(S) \neq \emptyset$, where $\operatorname{Fix}(T)$ and $\operatorname{Fix}(S)$ denote the set of fixed points of $T$ and $S$, respectively. Inspired by the 
work in [6] and [7], in this paper, we introduce the following split hierarchical monotone variational inclusion problem (SHMVIP):

$$
\text { Find } x^{*} \in \operatorname{Fix}(T) \text { such that } 0 \in f\left(x^{*}\right)+B_{1}\left(x^{*}\right) \text {, }
$$

and such that

$$
y^{*}:=A x^{*} \in \operatorname{Fix}(S) \text { solves } 0 \in g\left(y^{*}\right)+B_{2}\left(y^{*}\right) .
$$

We denote by $\Psi$ the set of solutions of the SHMVIP, that is,

$$
\Psi=\{x \text { solves }(1.8): A x \text { solves }(1.9)\}
$$

We propose an iterative algorithm to compute the approximate solutions of the SHMVIP. The weak convergence of the sequence generated by the proposed algorithm is studied. An example is presented to illustrate the proposed algorithm and result.

\section{Preliminaries}

Let $H$ be a real Hilbert space whose inner product and norm are denoted by $\langle\cdot, \cdot\rangle$ and $\|\cdot\|$, respectively. We denote by $x_{n} \rightarrow x$ (respectively, $x_{n} \rightarrow x$ ) the strong (respectively, weak) convergence of the sequence $\left\{x_{n}\right\}$ to $x$. Let $T: H \rightarrow H$ be an operator whose range is denoted by $R(T)$. The set of all fixed points of $T$ is denoted by $\operatorname{Fix}(T)$, that is, $\operatorname{Fix}(T)=$ $\{x \in H: x=T x\}$.

Definition 2.1 An operator $T: H \rightarrow H$ is said to be:

(a) nonexpansive if $\|T x-T y\| \leq\|x-y\|$, for all $x, y \in H$;

(b) strongly nonexpansive $[6,9,10]$ if $T$ is nonexpansive and

$$
\lim _{n \rightarrow \infty}\left\|\left(x_{n}-y_{n}\right)-\left(T x_{n}-T y_{n}\right)\right\|=0
$$

whenever $\left\{x_{n}\right\}$ and $\left\{y_{n}\right\}$ are bounded sequences in $H$ and $\lim _{n \rightarrow \infty}\left(\left\|x_{n}-y_{n}\right\|-\left\|T x_{n}-T y_{n}\right\|\right)=0$;

(c) averaged nonexpansive [11] if it can be written as

$$
T=(1-\alpha) I+\alpha S,
$$

where $\alpha \in(0,1), I$ is the identity operator on $H$, and $S: H \rightarrow H$ is a nonexpansive mapping;

(d) firmly nonexpansive if $\|T x-T y\|^{2} \leq\langle x-y, T x-T y\rangle$, for all $x, y \in H$;

(e) $\alpha$-inverse strongly monotone ( $\alpha$-ism) if there exists a constant $\alpha>0$ such that

$$
\langle T x-T y, x-y\rangle \geq \alpha\|T x-T y\|^{2}, \quad \text { for all } x, y \in H .
$$

The following example shows that every nonexpansive operator is not necessarily strongly nonexpansive. 
Example 2.1 Let $T:[-1,1] \rightarrow \mathbb{R}$ be defined by $T x=-x$, for all $x \in[-1,1]$. Then $T$ is nonexpansive but not strongly nonexpansive.

Indeed, let $x_{n}=1$ and $y_{n}=0$, for all $n$. Then $\left\{x_{n}\right\}$ and $\left\{y_{n}\right\}$ are bounded sequences. Also,

$$
\lim _{n \rightarrow \infty}\left|\left(x_{n}-y_{n}\right)-\left(T x_{n}-T y_{n}\right)\right|=\lim _{n \rightarrow \infty}|1+1|=2 \neq 0 .
$$

Thus, $T$ is not strongly nonexpansive.

The following result will be used in the sequel.

Proposition 2.1 [11] Let $T: H \rightarrow H$ be an operator.

(i) If $T$ is $v$-ism, then for $\gamma>0, \gamma T$ is $\frac{v}{\gamma}$-ism.

(ii) $T$ is averaged if and only if the complement $I-T$ is $v$-ism for some $v>\frac{1}{2}$. Indeed, for $\alpha \in(0,1), T$ is $\alpha$-averaged if and only if $I-T$ is $\frac{1}{2 \alpha}$-ism.

(iii) The composite of finitely many averaged mappings is averaged.

Let $\varphi: H \rightarrow H$ be a given single-valued $\alpha$-inverse strongly monotone operator and $\lambda \in$ $(0,2 \alpha)$. Then $(I-\lambda \varphi)$ is averaged. Indeed, since $\varphi$ is $\alpha$-inverse strongly monotone, $\lambda \varphi$ is $\frac{\alpha}{\lambda}$-ism. Thus, $I-\lambda \varphi$ is averaged as $\frac{\alpha}{\lambda}>\frac{1}{2}$.

Recall that a Banach space $X$ is said to satisfy Opial's condition [12] if whenever $\left\{x_{n}\right\}$ is a sequence in $X$ which converges weakly to $x$ as $n \rightarrow \infty$, then

$$
\limsup _{n \rightarrow \infty}\left\|x_{n}-x\right\|<\limsup _{n \rightarrow \infty}\left\|x_{n}-y\right\|, \quad \text { for all } y \in X, y \neq x .
$$

It is well known that every Hilbert space satisfies Opial's condition.

Lemma 2.1 (Demiclosedness principle) [12], Lemma 2 Let $C$ be a nonempty, closed, and convex subset of a real Hilbert space $H$ and $T: C \rightarrow C$ be a nonexpansive operator with $\operatorname{Fix}(T) \neq \emptyset$. If the sequence $\left\{x_{n}\right\} \subseteq C$ converges weakly to $x$ and the sequence $\left\{(I-T) x_{n}\right\}$ converges strongly to $y$, then $(I-T) x=y$. In particular, if $y=0$, then $x \in \operatorname{Fix}(T)$.

Let $B: H \rightrightarrows H$ be a set-valued mapping. The domain, range, and inverse of $B$ are denoted by

$$
D(B)=\{x \in H: B(x) \neq \emptyset\}, \quad R(B)=\bigcup_{x \in D(B)} B(x), \quad \text { and } \quad B^{-1}(y)=\{x \in H: y \in B(x)\},
$$

respectively.

Definition 2.2 The set-valued mapping $B: H \rightrightarrows H$ with nonempty values is said to be:

(a) monotone if

$$
\langle u-v, x-y\rangle \geq 0, \quad \text { for all } u \in B x, v \in B y \text {; }
$$

(b) maximal monotone if it is monotone and the graph

$$
G(B)=\{(x, u) \in H \times H: u \in B x\}
$$

of $B$ is not properly contained in the graph of any other monotone operator. 


\section{Algorithm and convergence result}

It is well known that when the set-valued mapping $B: H \rightrightarrows H$ is maximal monotone, then for each $x \in H$ and $\lambda>0$, there is a unique $z \in H$ such that $x \in(I+\lambda B) z[13,14]$. In this case, the operator $J_{\lambda}^{B}:=(I+\lambda B)^{-1}$ is called resolvent of $B$ with parameter $\lambda$. It is well known that $J_{\lambda}^{B}$ is a single-valued and firmly nonexpansive mapping.

Indeed, for any given $u \in H$, let $x, y \in J_{\lambda}^{B}(u)$. Then $x, y \in(I+\lambda B)^{-1}(u)$, and thus $u-x \in \lambda B x$ and $u-y \in \lambda B y$. The monotonicity of $\lambda B$ implies that

$$
\langle u-x-(u-y), x-y\rangle \geq 0
$$

This implies that $\|x-y\| \leq 0$, and thus $x=y$. Hence, $J_{\lambda}^{B}$ is single-valued.

Next we show that $J_{\lambda}^{B}$ is firmly nonexpansive mapping. For any $x, y \in H$, let $J_{\lambda}^{B}(x)=(I+$ $\lambda B)^{-1}(x)$ and $J_{\lambda}^{B}(y)=(I+\lambda B)^{-1}(y)$. This implies that $x \in(I+\lambda B)\left(J_{\lambda}^{B}(x)\right)$ and $y \in(I+\lambda B)\left(J_{\lambda}^{B}(y)\right)$. It follows that $\frac{1}{\lambda}\left(x-\left(J_{\lambda}^{B}(x)\right)\right) \in B\left(J_{\lambda}^{B}(x)\right)$ and $\frac{1}{\lambda}\left(y-\left(J_{\lambda}^{B}(y)\right)\right) \in B\left(J_{\lambda}^{B}(y)\right)$. The monotonicity of $B$ implies that

$$
\left\langle J_{\lambda}^{B}(x)-J_{\lambda}^{B}(y), \frac{1}{\lambda}\left(x-J_{\lambda}^{B}(x)\right)-\frac{1}{\lambda}\left(y-J_{\lambda}^{B}(y)\right)\right\rangle \geq 0,
$$

that is,

$$
\left\langle J_{\lambda}^{B}(x)-J_{\lambda}^{B}(y), x-y\right\rangle \geq\left\|J_{\lambda}^{B}(x)-J_{\lambda}^{B}(y)\right\|^{2} .
$$

Thus, $J_{\lambda}^{B}$ is firmly nonexpansive.

Let $\phi: H \rightarrow H$ be a given single-valued operator. Then

$$
0 \in \phi\left(x^{*}\right)+B\left(x^{*}\right) \quad \Leftrightarrow \quad x^{*} \in \operatorname{Fix}\left(J_{\lambda}^{B}(I-\lambda \phi)\left(x^{*}\right)\right) .
$$

Indeed, let $x^{*} \in \operatorname{Fix}\left(J_{\lambda}^{B}(I-\lambda \phi)\left(x^{*}\right)\right)$. Then $x^{*}=J_{\lambda}^{B}(I-\lambda \phi)\left(x^{*}\right)$. It follows that

$$
x^{*}=(I+\lambda B)^{-1}(I-\lambda \phi)\left(x^{*}\right) \quad \Leftrightarrow \quad x^{*}-\lambda \phi\left(x^{*}\right) \in(I+\lambda B)\left(x^{*}\right) \quad \Leftrightarrow \quad 0 \in \phi\left(x^{*}\right)+B\left(x^{*}\right) .
$$

Since $J_{\lambda}^{B}$ is firmly nonexpansive, and therefore, averaged. It is well known that the composition of averaged mapping is averaged, thus $J_{\lambda}^{B}(I-\lambda \varphi)$ is averaged. Since every averaged mapping is strongly nonexpansive [10], it follows that $J_{\lambda}^{B}(I-\lambda \varphi)$ is also strongly nonexpansive.

Let $B_{1}: H_{1} \rightrightarrows H_{1}$ and $B_{2}: H_{2} \rightrightarrows H_{2}$ be set-valued mappings with nonempty values, and let $f: H_{1} \rightarrow H_{1}$ and $g: H_{2} \rightarrow H_{2}$ be mappings. Let $T: H_{1} \rightarrow H_{1}$ and $S: H_{2} \rightarrow H_{2}$ be operators such that $\operatorname{Fix}(T) \neq \emptyset$ and $\operatorname{Fix}(S) \neq \emptyset$. Let $U:=J_{\lambda}^{B_{1}}(I-\lambda f)$ and $V:=J_{\lambda}^{B_{2}}(I-\lambda g)$. With the help of (3.1), (1.8), and (1.9) can be rewritten as

$$
\text { find } x^{*} \in \operatorname{Fix}(T) \text { such that } x^{*} \in \operatorname{Fix}\left(J_{\lambda}^{B_{1}}(I-\lambda f)\right) \text {, }
$$

and such that

$$
y^{*}:=A x^{*} \in \operatorname{Fix}(S) \text { solves } y^{*} \in \operatorname{Fix}\left(J_{\lambda}^{B_{2}}(I-\lambda g)\right) .
$$


Now we propose the following algorithm to compute the approximate solutions of the SHMVIP.

Algorithm 3.1 Initialization: Let $\lambda>0$ and take arbitrary $x_{0} \in H_{1}$.

ITERATIVE STEP: For a given current $x_{n} \in H_{1}$, compute

$$
x_{n+1}=T U\left(x_{n}+\gamma A^{*}(S V-I) A x_{n}\right)
$$

where $\gamma \in\left(0, \frac{1}{\|A\|^{2}}\right)$.

LAST STEP: Update $n:=n+1$.

Next we prove the weak convergence of the sequence generated by Algorithm 3.1.

Theorem 3.1 Let $A: H_{1} \rightarrow H_{2}$ be a bounded linear operator, $f: H_{1} \rightarrow H_{1}$ be an $\alpha_{1}$-inverse strongly monotone operator, $T: H_{1} \rightarrow H_{1}$ be a strongly nonexpansive operator such that $\operatorname{Fix}(T) \neq \emptyset, g: H_{2} \rightarrow H_{2}$ be an $\alpha_{2}$-inverse strongly monotone operator, $S: H_{2} \rightarrow H_{2}$ be a nonexpansive operator such that $\operatorname{Fix}(S) \neq \emptyset$, and $\alpha:=\min \left\{\alpha_{1}, \alpha_{2}\right\}$. Consider the operator $U:=J_{\lambda}^{B_{1}}(I-\lambda f)$ and $V:=J_{\lambda}^{B_{2}}(I-\lambda g)$ with $\lambda \in(0,2 \alpha)$, and $B_{1}: H_{1} \rightrightarrows H_{1}$ and $B_{2}: H_{2} \rightrightarrows H_{2}$ are two maximal monotone set-valued mappings with nonempty values. Then the sequence $\left\{x_{n}\right\}$ generated by Algorithm 3.1 converges weakly to an element $x^{*} \in \Psi$, provided $\Psi \neq \emptyset$.

Proof Let $p \in \Psi$. Then $T p=p, U p=p, S A p=A p$, and $V A p=A p$. Let $y_{n}:=x_{n}+\gamma A^{*}(S V-$ I) $A x_{n}$ and consider

$$
\begin{aligned}
\left\|y_{n}-p\right\|^{2}= & \left\|x_{n}+\gamma A^{*}(S V-I) A x_{n}-p\right\|^{2} \\
= & \left\|x_{n}-p\right\|^{2}+\gamma^{2}\left\|A^{*}(S V-I) A x_{n}\right\|^{2} \\
& +2 \gamma\left\langle x_{n}-p, A^{*}(S V-I) A x_{n}\right\rangle \\
\leq & \left\|x_{n}-p\right\|^{2}+\gamma^{2}\|A\|^{2}\left\|(S V-I) A x_{n}\right\|^{2} \\
& +2 \gamma\left\langle x_{n}-p, A^{*}(S V-I) A x_{n}\right\rangle .
\end{aligned}
$$

Consider the third term of inequality (3.5), we have

$$
\begin{aligned}
\left\langle x_{n}\right. & \left.-p, A^{*}(S V-I) A x_{n}\right\rangle \\
& =\left\langle A x_{n}-A p,(S V-I) A x_{n}\right\rangle \\
& =\left\langle(S V-I) A x_{n}-A p+A x_{n}-(S V-I) A x_{n},(S V-I) A x_{n}\right\rangle \\
& =\left\langle S V A x_{n}-A p, S V A x_{n}-A x_{n}\right\rangle-\left\|(S V-I) A x_{n}\right\|^{2} \\
& =\frac{1}{2}\left\|S V A x_{n}-A p\right\|^{2}+\frac{1}{2}\left\|S V A x_{n}-A x_{n}\right\|^{2}-\frac{1}{2}\left\|A x_{n}-A p\right\|^{2}-\left\|(S V-I) A x_{n}\right\|^{2} \\
& =\frac{1}{2}\left\|S V A x_{n}-S V A p\right\|^{2}+\frac{1}{2}\left\|S V A x_{n}-A x_{n}\right\|^{2}-\frac{1}{2}\left\|A x_{n}-A p\right\|^{2}-\left\|(S V-I) A x_{n}\right\|^{2} \\
& \leq \frac{1}{2}\left\|A x_{n}-A p\right\|^{2}+\frac{1}{2}\left\|S V A x_{n}-A x_{n}\right\|^{2}-\frac{1}{2}\left\|A x_{n}-A p\right\|^{2}-\left\|(S V-I) A x_{n}\right\|^{2} \\
& =-\frac{1}{2}\left\|(S V-I) A x_{n}\right\|^{2} .
\end{aligned}
$$


Combining (3.5) and (3.6), we obtain

$$
\begin{aligned}
\left\|y_{n}-p\right\|^{2} & \leq\left\|x_{n}-p\right\|^{2}+\gamma^{2}\|A\|^{2}\left\|(I-S V) A x_{n}\right\|^{2}-\gamma\left\|(S V-I) A x_{n}\right\|^{2} \\
& =\left\|x_{n}-p\right\|^{2}-\gamma\left(1-\gamma\|A\|^{2}\right)\left\|(S V-I) A x_{n}\right\|^{2} .
\end{aligned}
$$

Since $\gamma \in\left(0, \frac{1}{\|A\|^{2}}\right)$, we have $\gamma\left(1-\gamma\|A\|^{2}\right)>0$, and thus,

$$
\left\|y_{n}-p\right\| \leq\left\|x_{n}-p\right\|
$$

From the above inequality (3.7), we have

$$
\begin{aligned}
\left\|x_{n+1}-p\right\|^{2} & =\left\|T U y_{n}-T U p\right\|^{2} \\
& \leq\left\|U y_{n}-U p\right\|^{2} \\
& \leq\left\|y_{n}-p\right\|^{2} \\
& \leq\left\|x_{n}-p\right\|^{2}-\gamma\left(1-\gamma\|A\|^{2}\right)\left\|(S V-I) A x_{n}\right\|^{2} \\
& \leq\left\|x_{n}-p\right\|^{2} .
\end{aligned}
$$

This shows that $\left\|x_{n+1}-p\right\| \leq\left\|x_{n}-p\right\|$ and this implies that $\left\{\left\|x_{n}-p\right\|\right\}_{n=1}^{\infty}$ is a monotonic decreasing sequence, also $\left\{x_{n}\right\}$ is a bounded sequence, see [15], Theorem 4.5.10 and, hence, $\lim _{n \rightarrow \infty}\left\|x_{n}-p\right\|$ exists. Taking the limit at both sides in (3.9), and noticing that $\gamma(1-$ $\left.\gamma\|A\|^{2}\right)>0$, we have

$$
\lim _{n \rightarrow \infty}\left\|(S V-I) A x_{n}\right\|=0
$$

and since $y_{n}:=x_{n}+\gamma A^{*}(S V-I) A x_{n}$, we have $\left\|y_{n}-x_{n}\right\|=\gamma\|A\|\left\|(S V-I) A x_{n}\right\|$ thus in view of (3.10) we have

$$
\lim _{n \rightarrow \infty}\left\|y_{n}-x_{n}\right\|=0
$$

Since $\left\{x_{n}\right\}$ is a bounded sequence and it has a weakly convergent subsequence say, $x_{n_{i}} \rightarrow x^{*}$, [10] or with the help of Opial's condition [12], we can see that $x_{n} \rightarrow x^{*}$. Thus, we have $A x_{n} \rightarrow A x^{*}$. Since $S V$ is nonexpansive, from (3.10) and the closedness of $S V-I$ at 0 we obtain $S V A x^{*}=A x^{*}$. Next we show that $V A x^{*}=A x^{*}$. We have

$$
\left|\left\|S V A x_{n}-A p\right\|-\left\|A x_{n}-A p\right\|\right| \leq\left\|S V A x_{n}-A x_{n}\right\| .
$$

Taking the limit at both sides and by using (3.10), we obtain

$$
\begin{gathered}
\lim _{n \rightarrow \infty}\left|\left(\left\|S V A x_{n}-A p\right\|-\left\|A x_{n}-A p\right\|\right)\right|=0, \\
\left|\lim _{n \rightarrow \infty}\left(\left\|S V A x_{n}-A p\right\|-\left\|A x_{n}-A p\right\|\right)\right|=0, \\
\lim _{n \rightarrow \infty}\left(\left\|S V A x_{n}-A p\right\|-\left\|A x_{n}-A p\right\|\right)=0 .
\end{gathered}
$$


Since $S A p=A p$ and $V A p=A p$, by the nonexpansiveness of $S$ and $V$, we have

$$
\left\|S V A x_{n}-A p\right\| \leq\left\|V A x_{n}-A p\right\| \leq\left\|A x_{n}-A p\right\|,
$$

and therefore

$$
\left\|S V A x_{n}-A p\right\|-\left\|A x_{n}-A p\right\| \leq\left\|V A x_{n}-A p\right\|-\left\|A x_{n}-A p\right\| \leq 0 .
$$

Thus, we have

$$
\lim _{n \rightarrow \infty}\left(\left\|S V A x_{n}-A p\right\|-\left\|A x_{n}-A p\right\|\right) \leq \lim _{n \rightarrow \infty}\left(\left\|V A x_{n}-A p\right\|-\left\|A x_{n}-A p\right\|\right) \leq 0 .
$$

From (3.12) and (3.13), we obtain

$$
\lim _{n \rightarrow \infty}\left(\left\|V A x_{n}-A p\right\|-\left\|A x_{n}-A p\right\|\right)=0 .
$$

Since $V$ is averaged nonexpansive and every averaged nonexpansive map is strongly nonexpansive, we see that $V$ is strongly nonexpansive. Since $\{A p\}$ and $\left\{A x_{n}\right\}$ are bounded sequences, by the definition of strong nonexpansiveness of $V$, we have

$$
\lim _{n \rightarrow \infty}\left\|V A x_{n}-A x_{n}\right\|=0 .
$$

Since $V$ is nonexpansive, by the demiclosedness principle, we have

$$
V A x^{*}=A x^{*}
$$

Now, we show that $T x^{*}=x^{*}$ and $U x^{*}=x^{*}$. By using the nonexpansiveness of $T$ and $U$, in view of (3.4) and (3.8), we have

$$
0 \leq\left\|U y_{n}-p\right\|-\left\|T U y_{n}-p\right\| \leq\left\|y_{n}-p\right\|-\left\|T U y_{n}-p\right\| \leq\left\|x_{n}-p\right\|-\left\|x_{n+1}-p\right\| .
$$

This implies that

$$
\lim _{n \rightarrow \infty}\left(\left\|U y_{n}-p\right\|-\left\|T U y_{n}-p\right\|\right)=0 .
$$

From (3.8), we see that $\left\{y_{n}\right\}$ is a bounded sequence and thus $\left\{U y_{n}\right\}$ is bounded and since $\{p\}$ is a constant sequence thus $\{p\}$ is also bounded and since $T$ is strongly nonexpansive, we have

$$
\lim _{n \rightarrow \infty}\left\|U y_{n}-T U y_{n}\right\|=0
$$

In view of (3.4) and (3.8), by using the nonexpansiveness of $T U$, we have

$$
0 \leq\left\|y_{n}-p\right\|-\left\|T U y_{n}-p\right\| \leq\left\|x_{n}-p\right\|-\left\|x_{n+1}-p\right\| .
$$

It follows that

$$
\lim _{n \rightarrow \infty}\left(\left\|y_{n}-p\right\|-\left\|T U y_{n}-p\right\|\right)=0 .
$$


By using the nonexpansiveness of $T$ and $U$, we have

$$
\left\|T U y_{n}-p\right\| \leq\left\|U y_{n}-p\right\| \leq\left\|y_{n}-p\right\|
$$

and therefore

$$
\left\|T U y_{n}-p\right\|-\left\|y_{n}-p\right\| \leq\left\|U y_{n}-p\right\|-\left\|y_{n}-p\right\| \leq 0
$$

Thus, from (3.17), we have

$$
\lim _{n \rightarrow \infty}\left(\left\|U y_{n}-p\right\|-\left\|y_{n}-p\right\|\right)=0
$$

From (3.8) we see that $\left\{y_{n}\right\}$ is a bounded sequence and $\{p\}$ being a constant sequence, also bounded, by the strong nonexpansiveness of $U$, we have

$$
\lim _{n \rightarrow \infty}\left\|U y_{n}-y_{n}\right\|=0
$$

Next consider, for all $f \in H$,

$$
\begin{aligned}
\left\|f\left(y_{n}\right)-f\left(x^{*}\right)\right\| & =\left\|f\left(y_{n}\right)-f\left(x_{n}\right)+f\left(x_{n}\right)-f\left(x^{*}\right)\right\| \\
& \leq\left\|f\left(y_{n}\right)-f\left(x_{n}\right)\right\|+\left\|f\left(x_{n}\right)-f\left(x^{*}\right)\right\| \\
& \leq\|f\|\left\|y_{n}-x_{n}\right\|+\left\|f\left(x_{n}\right)-f\left(x^{*}\right)\right\| .
\end{aligned}
$$

Since $x_{n} \rightarrow x^{*}$ and from (3.11), we have $\lim _{n \rightarrow \infty}\left\|f\left(y_{n}\right)-f\left(x^{*}\right)\right\|=0$, thus $y_{n} \rightarrow x^{*}$. Thus, in view of (3.19) and by applying the demiclosedness principle, we have $U x^{*}=x^{*}$. Again, since $y_{n} \rightarrow x^{*}$, in view of (3.19), we have $U y_{n} \rightarrow x^{*}$. Thus, again in view of (3.16) and by applying the demiclosedness principle, we have $T x^{*}=x^{*}$.

Now, we illustrate Algorithm 3.1 and Theorem 3.1 by the following example.

Example 3.1 Let $H_{1}=H_{2}=H=\mathbb{R}$ and $B: H \rightrightarrows H$ be defined by

$$
B(x)= \begin{cases}\{1\}, & \text { if } x>0 \\ {[0,1],} & \text { if } x=0 \\ \{0\}, & \text { if } x<0\end{cases}
$$

Then, as shown in [16], $B$ is a set-valued maximal monotone mapping. We define the mappings $A, f, h, T, S: H \rightarrow H$ by

$$
\begin{aligned}
& A x=\frac{x}{2}, \quad \text { for all } x \in H, \\
& f x=h x=\frac{2 x}{3}, \quad \text { for all } x \in H, \\
& T x=\frac{x}{3}, \quad \text { for all } x \in H,
\end{aligned}
$$


Table 1 The values of $\left\{x_{n}\right\}$ with initial guess $x_{1}=10, x_{1}=15$, and $x_{1}=20$

\begin{tabular}{lllllllllll}
\hline $\boldsymbol{n}$ & $\mathbf{1}$ & $\mathbf{2}$ & $\mathbf{3}$ & $\mathbf{4}$ & $\mathbf{5}$ & $\mathbf{6}$ & $\mathbf{7}$ & $\mathbf{8}$ & $\mathbf{9}$ & $\mathbf{1 0}$ \\
\hline$x_{n}$ & 10 & 0.7037 & 0.0495 & 0.0035 & 0.0002 & 0.0000 & 0.0000 & 0.0000 & 0.0000 & 0.0000 \\
$x_{n}$ & 15 & 1.0556 & 0.0743 & 0.0052 & 0.0004 & 0.0000 & 0.0000 & 0.0000 & 0.0000 & 0.0000 \\
$x_{n}$ & 20 & 1.4074 & 0.0990 & 0.0070 & 0.0005 & 0.0000 & 0.0000 & 0.0000 & 0.0000 & 0.0000 \\
\hline
\end{tabular}

and

$$
S x=\frac{4 x}{5}, \quad \text { for all } x \in H
$$

respectively. It is easy to show that $A$ is a bounded linear operator, $f$ and $h$ are $\frac{1}{3}$-ism, $T$ is firmly nonexpansive, and thus $T$ is strongly nonexpansive [10] and $S$ is nonexpansive. Let $B_{1}(x)=B_{2}(x)=B x$. Then $B_{1}$ and $B_{2}$ are maximal monotone set-valued mappings. Let $J_{\lambda}^{B_{1}}(x)=J_{\lambda}^{B_{2}}(x)=\frac{x}{2}$ be the resolvent operator. The values of $\left\{x_{n}\right\}$ with different values of $n$ are reported in the Table 1 . All codes were written in Matlab R2010.

Table 1 shows that the sequence $\left\{x_{n}\right\}$ converges to 0 , which is the required solution.

\section{Competing interests}

The authors declare that they have no competing interests.

\section{Authors' contributions}

All authors contributed equally to the writing of this paper. All authors read and approved the final manuscript.

Received: 10 March 2015 Accepted: 26 June 2015 Published online: 18 July 2015

\section{References}

1. Censor, Y, Gibali, A, Reich, S: Algorithms for the split variational inequality problem. Numer. Algorithms 59, $301-323$ (2012)

2. Ansari, QH, Lalitha, CS, Mehta, M: Generalized Convexity, Nonsmooth Variational Inequalities and Nonsmooth Optimization. CRC Press, Boca Raton (2014)

3. Censor, Y, Elfving, Y: A multiprojection algorithm using Bregman projection in product space. Numer. Algorithms 8 , 221-239 (1994)

4. Ansari, QH, Rehan, A: Split feasibility and fixed point problems. In: Ansari, QH (ed.) Nonlinear Analysis: Approximation Theory, Optimization and Applications, pp. 281-322. Birkhäuser, Basel (2014)

5. Byrne, C: Iterative oblique projection onto convex subsets and the split feasibility problem. Inverse Probl. 18, 441-453 (2002)

6. Ansari, QH, Nimana, N, Petrot, N: Split hierarchical variational inequality problems and related problems. Fixed Point Theory Appl. 2014, Article ID 208 (2014)

7. Moudafi, A: Split monotone variational inclusions. J. Optim. Theory Appl. 150, 275-283 (2011)

8. Zeidler, E: Nonlinear Functional Analysis and Its Applications III: Variational Methods and Optimization. Springer, Berlin (1984)

9. Bruck, RE, Reich, S: Nonexpansive projections and resolvent of accretive operators in Banach spaces. Houst. J. Math. 3 459-470 (1977)

10. Cegeilski, A: Iterative Methods for Fixed Point Problems in Hilbert Spaces. Springer, New York (2012)

11. Byrne, C: A unified treatment of some iterative algorithms in signal processing and image reconstruction. Inverse Probl. 20, 103-120 (2004)

12. Opial, Z: Weak convergence of the sequence of successive approximations for nonexpansive mappings. Bull. Am. Math. Soc. 73, 591-597 (1976)

13. Sahu, DR, Ansari, QH: Hierarchical minimization problems and applications. In: Ansari, QH (ed.) Nonlinear Analysis: Approximation Theory, Optimization and Applications. Birkhäuser, Basel (2014)

14. Takahashi, W: Nonlinear Functional Analysis, Fixed Point Theory and Its Applications. Yokohama Publishers, Yokohama (2000)

15. Borwein, JM, Zhu, QJ: Techniques of Variational Analysis. Springer, Berlin (2005)

16. Phelps, RR: Lectures on Maximal Monotone Operators, pp. 15-28. Dept. Math. GN-50, Univ. of Wash., Prague/Paseky Summer School, Czech Republic (1993) 